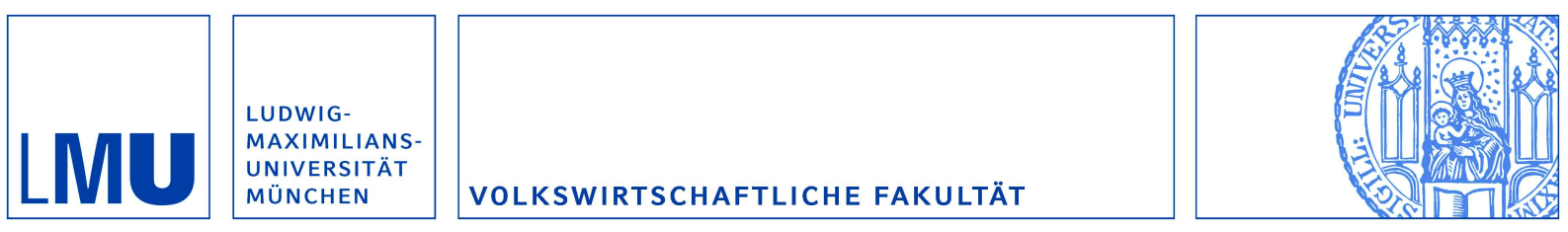

Baver, Christian:

The Reservation Wage under CARA and Limited Borrowing

Munich Discussion Paper No. 2009-8

Department of Economics

University of Munich

Volkswirtschaftliche Fakultät

Ludwig-Maximilians-Universitäł München

Online at https://doi.org/10.5282/ubm/epub. 10291 


\title{
The Reservation Wage under CARA and Limited Borrowing
}

\author{
Christian Bauer*
}

A continuous-time sequential job search model with savings and CARA preferences is solved analytically without resorting to unlimited borrowing and real-valued consumption. I isolate the effects of limited borrowing and nonnegative consumption as well as risk-aversion on the reservation wage by using a system of ordinary differential equations.

JEL Classification: C61, E21, D91, J64.

Keywords: labor income risk, wealth-dependent reservation wage, borrowing limit.

\section{Introduction}

Motivated by considerable academic and popular interest in wealth-dependent unemployment policies, I study the impact of a borrowing limit on the optimal behavior of a risk-averse worker in a continuoustime sequential job search model with savings. Deviating from expected income maximization makes it difficult to characterize optimal consumption and the reservation wage analytically in this environment. Recent research employs a very specific set of assumptions: with constant absolute risk aversion (CARA), unlimited borrowing, and no nonnegativity constraint on consumption, a closed-form solu-

*Ludwig-Maximilians-University Munich. Kaulbachstr. 45, 80539 Munich, Germany. Phone: ++49-89-2180-3193. Email: christian.bauer@lrz.uni-muenchen.de. Financial support from the Deutsche Forschungsgemeinschaft through GRK 801 is gratefully acknowledged. I thank Klaus Wälde for his encouragement, comments, and suggestions. I also thank Andrea Schrage, Lutz Arnold, Alfred Maußner, Nicolas Sauter, and Stefanie Trepl for helpful discussions and comments. Any remaining errors are, of course, my own responsibility. This version: March 2009. 
tion exists (see, e.g., Acemoglu and Shimer, 1999, Shimer and Werning, 2007, 2008). This solution includes the following properties: wealth enters consumption linearly, wealth does not affect the reservation wage, and wealth and consumption fall below any real number with positive probability. In this paper, I solve the model without imposing a linear solution by using a two-dimensional system of ordinary differential equations. It then is possible to analytically dissect the effects of lower bounds on wealth and consumption as well as risk-aversion on optimal consumption and the reservation wage. Limited borrowing increases the marginal propensity to consume out of wealth as wealth declines, thereby causing a falling sequence of reservation wages over the unemployment spell.

\section{Model and Solution}

Consider a continuous-time partial equilibrium model of sequential job search with savings. An unemployed worker receives job offers with random wage draws $w$ at Poisson rate $\alpha .{ }^{1}$ There is no job separation and no on-the-job search. The individual maximizes her expected lifetime utility at time $t$,

$$
U(t)=E_{t} \int_{t}^{\infty} e^{-\rho(\tau-t)} u(c(\tau)) d \tau
$$

where $u(c)=-e^{-\gamma c}$ is the instantaneous utility from consumption $c$. The discount rate $\rho$ equals the uniform interest rate on savings and debt, $r$. The unemployed individual receives constant benefits $b$. Using consumption as the numéraire, her asset holdings evolve according to

$$
\dot{a}^{u}=r a+b-c^{u} .
$$

When employed, the worker lives in a stationary world. Hence, $c^{e}=r a+w, \dot{a}^{e}=0$, and the value of being employed is $V^{e}(w, a)=\frac{u\left(c^{e}(w, a)\right)}{r}$ (see Appendix 4.A). When unemployed, the worker chooses consumption and a reservation wage to maximize (1) subject to (2) and a no-Ponzi game condition

\footnotetext{
${ }^{1}$ Under CARA, recall of offers is not optimal and can be ignored.
} 
that limits the growth rate of debt to $r$ (at this stage $a, c \in \mathbb{R}$ ).

\section{Deterministic Wage Offer}

Suppose that there is only a single deterministic wage offer $w$. Before turning to the general solution, I follow Shimer and Werning (2008) and derive a closed-form solution by going through a verification theorem (cf. Merton, 1969, 1971, among others). If an optimal Markov control exists, the HamiltonJacobi-Bellman (HJB) equation is $\rho V^{u}(a)=\max _{c^{u}}\left[u\left(c^{u}\right)+\left(r a+b-c^{u}\right) V^{u \prime}(a)\right]$, where $V^{u}(a)$ is the value of being unemployed when holding $a$ units of wealth. The first order condition (f.o.c.) for consumption, $u^{\prime}\left(c^{u}\right)=V^{u \prime}(a)$, defines the policy function $c^{u}=c^{u}(a)$. We easily verify that the linear guesses ( $g$ for guess and the $\delta_{i}$ 's are constants) $c_{g}^{u}=\delta_{0} a+\delta_{1}$ and $V_{g}^{u}(a)=\delta_{2} u\left(c_{g}^{u}\right)$ satisfy both the f.o.c. and the HJB equation if $\delta_{0}=\frac{1}{\delta_{2}}=r$ and $\delta_{1}$ solves $\left(b-\delta_{1}\right) \frac{\gamma r}{\alpha}=e^{-\gamma\left(w-\delta_{1}\right)}-1$, which determines a unique $b<\delta_{1}^{\star}<w .^{2}$ We will see below that $\delta_{1}^{\star}$, i.e. the fraction of labor income used to finance consumption, is equal to the reservation wage in the case where wages are drawn from a distribution and there is no lower bound on wealth and consumption. This linear solution (which e.g. corresponds to Shimer and Werning, 2008, without a wage distribution) implies $\dot{a}^{u}=r a+b-\left(r a+\delta_{1}^{\star}\right)=b-\delta_{1}^{\star}<0$. Wealth falls during the unemployment spell and consumption declines along with it. Consumption becomes negative at $a=-\frac{\delta_{1}^{\star}}{r}$. If we impose ad hoc that consumption must be nonnegative, debt still continues to grow if it reaches $\frac{\delta_{1}^{\star}}{r}$ (if $c^{u}=0, \dot{a}^{u}=r a+b<0$ for $a<-\frac{b}{r}$ ). Debt exceeds any real number with positive probability.

We now turn to the general solution. That is, we describe the optimal behavior of an unemployed worker by a two-dimensional system of differential equations. The first equation is given by the law of

\footnotetext{
${ }^{2}$ The left hand side falls in $\delta_{1}$, intersecting the horizontal axis at $\delta_{1}=b$ from above. The right hand side is monotonically increasing in $\delta_{1}$, equals $e^{-\gamma w}$ at $\delta_{1}=0$, and intersects the horizontal axis at $\delta_{1}=w$ from below.
} 


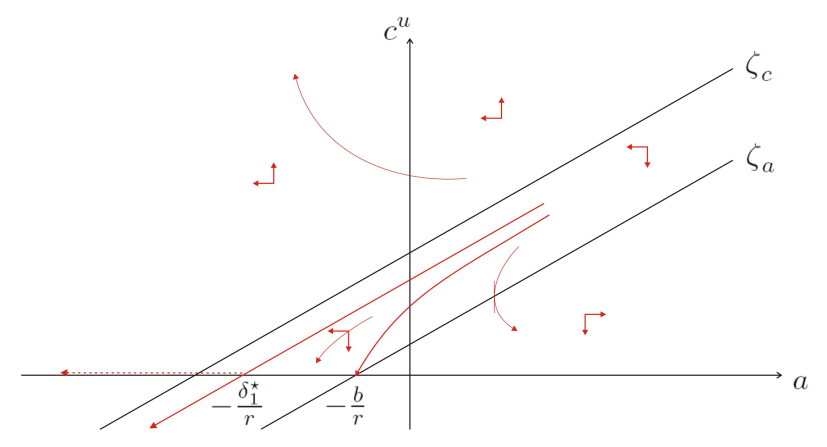

Figure 1: Consumption and Wealth Dynamics

motion for wealth in (2). The second equation is given by the evolution of optimal consumption (see Appendix 4.B):

$$
\dot{c}^{u}=-\frac{\alpha}{\gamma}\left[1+u\left(r a+w-c^{u}\right)\right] .
$$

The dynamics of the system are illustrated in Figure 1. Wealth is constant on $\zeta_{a}(a) \equiv r a+b$. Consumption is constant on $\zeta_{c}(a) \equiv r a+w$ and on $\zeta_{a}(a)$. Consumption and wealth diverge from $\zeta_{c}$ and $\zeta_{a}$, respectively. Hence, the optimal path lies between $\zeta_{a}(a)$ and $\zeta_{c}(a)$ (consumption is a normal good). ${ }^{3}$ The closed-form solution above is the trajectory consistent with the terminal condition $c^{u}\left(-\frac{\delta_{1}^{\star}}{r}\right)=0$. The dashed line depicts the solution including a nonnegativity constraint on consumption. Now suppose that wealth must not fall below some lower bound $\bar{a}$. Such a borrowing limit provides a globally saddle-path stable steady state at $(c, a)=\left(\zeta_{a}(\bar{a}), \bar{a}\right)$. The policy function must then be strictly increasing and strictly concave in wealth, see Figure 1 (where $\bar{a} \equiv-\frac{b}{r}$ ). Given initial asset holdings $a(0) \geq \bar{a}$, consumption starts on this trajectory and declines towards the steady state. If the unemployed does not leave unemployment, her consumption level reaches $\zeta_{a}(\bar{a})(0$ if $\bar{a}=-\frac{b}{r}$ ) in finite time, and then remains constant until she finds a job. Intuitively, limited borrowing rules out debt levels which are implied by a linear solution while leaving consumption of very wealthy

\footnotetext{
${ }^{3}$ Above $\zeta_{c}(a), c^{u} \rightarrow \infty$ while $a \rightarrow-\infty$. Below $\zeta_{a}(a), c^{u} \rightarrow-\infty$ while $a \rightarrow \infty$. Neither case can be part of a feasible optimal program.
} 
individuals virtually unaffected.

\section{Random Wage Offers}

With this background, consider wage offers drawn from a distribution $F(w)$ with support $[0, \infty)$ and $F^{\prime}(w)>0$ for some $w>0$. This gives rise to a reservation wage $\bar{w}$ that leaves the individual indifferent between accepting the job and remaining unemployed: $V^{e}(a, \bar{w}) \equiv V^{u}(a)$. Implicit differentiation using $V^{e}(a, w)=\frac{u(r a+w)}{r}(w \geq \bar{w})$ and $V^{u \prime}(a)=u^{\prime}\left(c^{u}(a)\right)$ shows

$$
\frac{d \bar{w}}{d a}=\left[\frac{u^{\prime}\left(c^{u}(a)\right)}{u^{\prime}\left(c^{e}(a, \bar{w})\right)}-1\right] r
$$

The reservation wage is independent of wealth if $c^{u}(a)=c^{e}(a, \bar{w})$ and increasing in wealth if $c^{u}(a)<c^{e}(a, \bar{w})$. If the model is solved without lower bounds on wealth and consumption, guessing and verifying a linear policy function in fact yields $c^{u \star}(a)=r a+\bar{w}^{\star}=c^{e}\left(\bar{w}^{\star}, a\right)$ (Shimer and Werning, 2008, Proposition 1), so that $\frac{d \bar{w}}{d a}=0$. As shown in Appendix 4.A, the reservation wage is then determined by

$$
\bar{w}^{\star}=b+\frac{\alpha}{\gamma r} \int_{\bar{w}^{\star}}^{\infty}\left[1+u\left(w-\bar{w}^{\star}\right)\right] d F(w) \quad(>b) .
$$

Implicit differentiation proves that $\bar{w}^{\star}$ decreases uniformly in the Arrow-Pratt measure of absolute

risk aversion at all wealth levels, $\frac{\partial \bar{w}^{\star}}{\partial \gamma}<0$. Analogously to the previous section, $\bar{w}^{\star}>b$ implies $-a>x$ for all $x>0$ with positive probability. Lower bounds on wealth and consumption require optimal consumption to be non-linear and turn the reservation wage into a function of wealth.

To prove this assertion, consider again the evolution of optimal consumption (see Appendix 4.B):

$$
\dot{c}^{u}=-\frac{\alpha}{\gamma} \int_{\bar{w}(a)}^{\infty}\left[1-\frac{u^{\prime}\left(c^{e}(a, w)\right)}{u^{\prime}\left(c^{u}(a)\right)}\right] d F(w) .
$$


Using $u^{\prime}(c)=-\gamma u(c)$ and $c^{e}=r a+w$ in (6), consumption is constant on

$$
c_{0}^{u}(a)=r a+(u)^{-1}\left(\int_{\bar{w}(a)}^{\infty} u(w) \frac{d F(w)}{1-F(\bar{w}(a))}\right),
$$

i.e. if the instantaneous income from benefits and dissaving $\left(b-\dot{a}=c^{u}-r a\right)$ equals the certainty equivalent of the lottery over future wages. Consumption is also constant on the $\dot{a}=0$ locus, which remains unchanged.

Proposition. Suppose $a \geq \bar{a} \equiv-\frac{b}{r}$ and $c \geq 0$. Then, consumption, wealth, and the reservation wage are strictly declining over the unemployment spell until the stationary point $(c, a)=(0, \bar{a})$ is reached. The reservation wage (which is increasing in wealth) exceeds the unemployment benefits at all wealth levels.

The proof is in Appendix 4.C. To interpret this finding, notice that an increase in unemployment

benefits raises $c^{u \star}$ one-by-one $\left(\frac{\partial c^{u \star}}{\partial \bar{w}^{\star}} \frac{\partial \bar{w}^{\star}}{\partial b}=1\right)$. With sufficient liquidity, unemployment benefits provide a strong incentive to remain unemployed, waiting for better offers. Limited access to borrowing mitigates this "benefit-to-mouth" effect and increases the need to accept a job as wealth declines.

\section{Conclusion}

We solved a sequential job search model with savings and CARA preferences without resorting to unlimited borrowing and real-valued consumption. In the benchmark case where $a, c \in \mathbb{R}$, consumption is linear and changes in unemployment benefits and absolute risk aversion affect consumption equally at all wealth levels. Holding absolute risk aversion constant, limited borrowing mitigates the impact of unemployment benefits on current consumption, and leads to a declining sequence of reservation wages over the unemployment spell. 


\section{Appendix}

\section{A Optimal Behavior}

The value function of an employed worker is $r V^{e}(a)=\max _{c^{e}}\left\{u\left(c^{e}\right)+\left[r a+w-c^{e}\right] V^{e \prime}(a)\right\}$. The f.o.c. reads $u^{\prime}\left(c^{e}\right)=V^{e \prime}(a)$. The "educated guesses" $c_{g}^{e}=\gamma_{0} a+\gamma_{1}$ and $V_{g}^{e}(a)=\gamma_{2} u\left(c_{g}^{e}\right)$ solve the f.o.c. and the HJB equation if $\gamma_{0} \gamma_{2}=1$ and $\frac{r}{\gamma_{0}}=1-\left[\left(r-\gamma_{0}\right) a+w-\gamma_{1}\right] \gamma_{2}$. As wealth drops out for $\gamma_{0}=r, c^{e}=r a+w$ and $V^{e}=\frac{u\left(c^{e}\right)}{r}$ (a naturally exceeds the lowest admissible wealth level). If $a, c \in \mathbb{R}$, the unemployed's HJB equation $\rho V^{u}=$ $\max _{c^{u}}\left\{u\left(c^{u}\right)+\left(r a+b-c^{u}\right) V^{u \prime}(a)+\alpha \int_{0}^{\infty} \max \left[V^{e}(a, w)-V^{u}(a), 0\right] d F(w)\right\}$ and f.o.c., $V^{e}=\frac{u\left(c^{e}\right)}{r}$, and $V^{e}(\bar{w}, a) \equiv V^{u}(a)$ analogously verify $c^{u}=\kappa_{0} a+\kappa_{1}, V^{u}(a)=\kappa_{2} u\left(c^{u}(a)\right)$ for $\kappa_{0}=\frac{1}{\kappa_{2}}=r$ and $\kappa_{1}=\bar{w}^{\star}$. Substituting these expressions and the f.o.c. in the HJB equation delivers (5).

\section{B Evolution of Optimal Consumption}

Substituting $u^{\prime}\left(c^{u}\right)=V^{u \prime}(a)$ in the unemployed's HJB equation, differentiating, and using the f.o.c., $r=\rho$, and $-\alpha\left[V^{e}(a, \bar{w}(a))-V^{u}(a)\right] \bar{w}^{\prime}(a)=0$ gives

$$
\left[r a+b-c^{u}(a)\right] V^{u \prime \prime}(a)=-\alpha \int_{\bar{w}(a)}^{\infty}\left[V^{e \prime}(a, w)-V^{u \prime}(a)\right] d F(w) .
$$

Differentiating $V^{u \prime}(a)$ using the Change of Variable Formula (CVF, cf. Sennewald and Wälde, 2006, Sennewald, 2007a,b, Wälde, 2008, Øksendal, 2003) and canceling $1-F(\bar{w}(a))$ yields

$$
d V^{u \prime}(a)=V^{u \prime \prime}(a) d a^{u}+\left[\int_{\bar{w}(a)}^{\infty}\left[V^{e \prime}(a, w)-V^{u \prime}(a)\right] d F(w)\right] d q_{\alpha}
$$

where $d q_{\alpha}$ is the increment of the Poisson process. Substituting with the f.o.c.'s in both employment states, $d a^{u}=\left(r a+b-c^{u}\right) d t$, and using the resulting expression in (A.1) $\left(d q_{\alpha}=0\right.$ for an individual who does not find a job), we get

$$
d u^{\prime}\left(c^{u}\right)=-\alpha \int_{\bar{w}(a)}^{\infty}\left[u^{\prime}\left(c^{e}\right)-u^{\prime}\left(c^{u}\right)\right] d F(w) d t .
$$


Let $v \equiv\left(u^{\prime}\left(c^{u}\right)\right)^{-1}$ such that $d v=d c^{u}$. Applying the CVF to $v$ yields $d v\left(\left(u^{\prime}(c)\right)=\right.$ $v^{\prime}\left(u^{\prime}(c)\right) d u^{\prime}\left(c^{z}(a)\right) d t+\left[v\left(u^{\prime}\left(c^{u}\right)\right)-v\left(u^{\prime}\left(c^{e}\right)\right)\right] d q_{\alpha}$. Using $d v=d c^{u}, v^{\prime}=u^{\prime \prime}\left(c^{u}(a)\right)^{-1}, d q_{\alpha}=0$, and $d u^{\prime}\left(c^{u}\right)$ from (A.2), we find $\dot{c}^{u}=-\frac{\alpha}{u^{\prime \prime}\left(c^{u}\right)} \int_{\bar{w}(a)}^{\infty}\left[u^{\prime}\left(c^{e}\right)-u^{\prime}\left(c^{u}\right)\right] d F(w)$. Inserting $u^{\prime \prime}\left(c^{u}\right)=-\gamma u^{\prime}\left(c^{u}\right)$ and $\frac{u^{\prime}\left(c^{e}\right)}{u^{\prime}\left(c^{u}\right)}=-u\left(c^{e}-c^{u}\right)$ gives $(3)$.

\section{C Proof of the Proposition}

In three steps. (a) $c_{0}^{u}(a)>\zeta_{a}(a)$ and hence $\dot{c}^{u}(a)<0, \dot{a}^{u}<0$ for all $a>\bar{a}$; (b) $c^{u}(a)>r a+b$ $\operatorname{implies} c^{e}(a, \bar{w}(a))>c^{u}(a) ;(\mathbf{c}) \bar{w}(a)>b$.

(a) follows from i) $c^{u}(a) \neq r a+b$ for $a>\bar{a}$ and ii) $c^{u}(a)>r a+b, \dot{c}^{u}(a)<0$, and $\dot{a}^{u}<0$ for $a$ slightly above $\bar{a}$ since $c_{0}^{u}(\bar{a})>\zeta_{a}(\bar{a})=0$. i) If $c^{u}\left(a^{\prime}\right)=r a^{\prime}+b, \dot{a}^{u}=0$ and thus $\dot{c}^{u}\left(a^{\prime}\right)=0$. Then, (7) implies $u^{\prime}(b)<\frac{\int_{b}^{\infty} u^{\prime}(w) d F(w)}{1-F(b)}$, a contradiction since (6) requires equality if $\dot{c}^{u}=0$. ii) $c^{u}(\bar{a})=0$ since $c^{u}(a) \geq 0$ and $c^{u}(\bar{a})>0$ implies $\dot{a}^{u}<0$ at $a=\bar{a}$. From $c^{u}(\bar{a})=0$ and $(4)$, $\bar{w}(\bar{a})>,=,<b$ if and only if $\bar{w}^{\prime}(\bar{a})>,=,<b \cdot \bar{w}(\bar{a})<b$ violates $a \geq \bar{a}$. By assumption, $F^{\prime}(w)>0$ for some $w>\bar{w}(\bar{a})$. Hence, $u^{\prime \prime}<0$ and $(7)$ imply $\frac{\int_{\bar{w}(\bar{a})}^{\infty} u^{\prime}(\bar{w}(\bar{a})-b) d F(w)}{1-F(\bar{w}(\bar{a}))}>\frac{\int_{\bar{w}(\bar{a})}^{\infty} u^{\prime}(w-b) d F(w)}{1-F(\bar{w}(\bar{a}))}=u^{\prime}\left(c_{0}^{u}(\bar{a})\right)$. Accordingly, $u^{\prime}(\bar{w}(\bar{a})-b)>u^{\prime}\left(c_{0}^{u}(\bar{a})\right)$ so that, from $u^{\prime \prime}<0$ and $\bar{w}(\bar{a}) \geq b, c_{0}^{u}(\bar{a})>\bar{w}(\bar{a})-b \geq 0$. Hence, $c_{0}^{u}(\bar{a})>\zeta_{a}(\bar{a})$. Continuity of $c_{0}^{u}(a)$ and the instability of $c_{0}^{u}(a)$ and $\zeta_{a}(a)$ complete the proof.

(b) Since the $\dot{c}^{u}=0$ locus is unstable, and $\dot{a}^{u}<0$ is implied by $c^{u}(a)>r a+b$, we have $c^{u}(a)<c_{0}^{u}(a) \cdot u^{\prime \prime}<0$ thus yields $u^{\prime}\left(c^{u}(a)\right)>u^{\prime}\left(c_{0}^{u}(a)\right)=\frac{\int_{\bar{w}(a)}^{\infty} u^{\prime}\left(c^{e}(a, w)\right) d F(w)}{1-F(\bar{w}(a))}$. This implies $u^{\prime}\left(c^{u}(a)\right)[1-F(\bar{w}(a))]>\int_{\bar{w}(a)}^{\infty} u^{\prime}\left(c^{e}(a, w)\right) d F(w)>\int_{\bar{w}(a)}^{\infty} u^{\prime}\left(c^{e}(\bar{w}(a), a)\right) d F(w)$, i.e. $u^{\prime}\left(c^{u}(a)\right)>$ $u^{\prime}\left(c^{e}(\bar{w}(a), a)\right)$

(c) $\bar{w}(\bar{a}) \geq b$ (from the proof of (a)), (4), and (b) imply $\bar{w}(a)>b$ for $a>\bar{a}$. Suppose $\bar{w}(\bar{a})=b$. Then, $V^{e}(\bar{w}, a)=V^{u}(a)$ and $V^{e}(w, a)=\frac{u(r a+w)}{r}$ imply $r V^{u}(\bar{a})=u(0)$. Substituting this in the maximized HJB equation of the unemployed evaluated at $\bar{a}$ using $c^{u}(\bar{a})=0$ (see (a)) and $\rho=r$ 
gives $\int_{b}^{\infty}\left[V^{e}(w, \bar{a})-V^{u}(\bar{a})\right] d F(w) \int_{b}^{\infty}[1+u(w-b)] d F(w)=0$, a contradiction since $F^{\prime}(w)>0$ for some $w>b$ and $u(0)=-1$.

\section{References}

Acemoglu, D., And R. Shimer (1999): "Efficient Unemployment Insurance," Journal of Political Economy, 107(5), 893-928.

Merton, R. C. (1969): "Lifetime Portfolio Selection under Uncertainty: The Continuous-Time Case," Review of Economics and Statistics, 51(3), 247-257.

(1971): "Optimum Consumption and Portfolio Rules in a Continuous-Time Model," Journal of Economic Theory, 3(4), 373-413.

Øksendal, B. (2003): Stochastic Differential Equations: An Introduction with Applications. Springer.

Sennewald, K. (2007a): "Controlled Stochastic Differential Equations under Poisson Uncertainty and with Unbounded Utility," Journal of Economic Dynamics and Control, 31(4), 1106-1131.

(2007b): Stochastic Control, Optimal Saving, and Job Search in Continuous Time. Dissertation, TU Dresden, Germany.

Sennewald, K., And K. WÄLde (2006): "Itô's Lemma' and the Bellman Equation for Poisson Processes: An Applied View," Journal of Economics, 89(1), 1-36.

Shimer, R., And I. Werning (2007): "Reservation Wages and Unemployment Insurance," The Quarterly Journal of Economics, 122(3), 1145-1185.

(2008): "Liquidity and Insurance for the Unemployed," American Economic Review, 98(5), 1922-1942.

WäLDE, K. (2008): Applied Intertemporal Optimization. www.waelde.com/aio. 